\title{
Vieillir avec humour et philosophie
}

\section{Jean Martin}

Dr med., membre de la rédaction

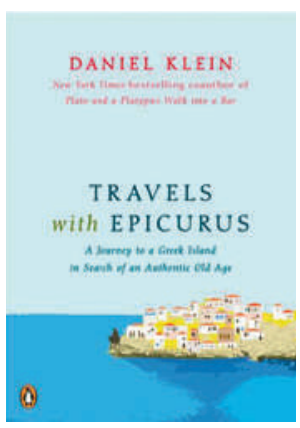

Daniel Klein

Travels with Epicurus

A Journey to a Greek Island in Search

of an Authentic Old Age

New York: Penguin Books; 2012.

163 pages. $9.50 \mathrm{CHF}$.

ISBN 978-0143121930

Daniel Klein est un écrivain américain de la côte Est, auteur de romans policiers et à suspense et d'ouvrages traitant de philosophie. Deux livres en particulier ont rencontré le succès: Plato and a Platypus Walk into a Bar en 2007, et en 2012 celui dont je parle ici'. L'auteur est né en 1939, nous sommes contemporains et c'est un ami du même âge qui m'a recommandé ce livre - recommandation qu'après lecture je passe plus loin.

Après une vie professionnelle très active, Klein est décidé à bien vivre sa vieillesse, par quoi il faut entendre qu'il n'est pas du nombre des «forever youngsters» (fréquents aux Etats-Unis), qui veulent à tout prix, au-delà de 70 ans, continuer à vivre et se comporter comme des jeunes (multiples poursuites, sport, sexualité). Non, il entend accepter l'âge et ses limites; profiter au mieux de ce que propose la vie quand on est senior, sans singer d'autres périodes de l'existence. Pour alimenter sa réflexion, il se penche sur les écrits de philosophes: les anciens Grecs et tout particulièrement Epicure ${ }^{2}$, Platon et Aristote, ainsi que les stoïciens romains, Montaigne et des modernes comme Heidegger. Il s'intéresse aux philosophies asiatiques. Tout cela avec humour. «Les vieilles personnes se déplacent lentement. Chez nous, le terrain rocailleux est interne - os fragiles, muscles défaillants, cœurs affaiblis [...]. Je réalise que, d'habitude, je luttais pour accélérer mon pas trop tranquille. Cependant, aujourd'hui, il me semble très clair que la lenteur a des vertus extraordinaires.» Epicure recommande, quand l'âge est venu, de laisser derrière soi «la prison du monde du commerce, des affaires et de la politique», ce qui, dit Klein, libère et permet de concentrer la réflexion sur d'autres sujets, souvent plus intimes - et de ne plus avoir peur du futur. A propos de ce que les gens âgés voudraient réaliser encore, il observe que «la nouveauté elle-même devient vieille. En visitant le douzième endroit dans le monde où il faut avoir été avant de mourir, voir de l'exotique est devenu moins attirant, vous en avez déjà vu onze fois.» Oscar Wilde: "Dans ce monde, il n'y a que deux tragédies. L'une est de ne pas obtenir ce que l'on désire, l'autre est de l'obtenir.» Klein cite Erik Erikson pour qui il s'agit «d'arriver à un sage sens d'accomplissement, de plénitude et à une acceptation sereine de soi-même, malgré les erreurs et faux pas faits sur la route».

Se souvenir de ce que l'on a: «L'expérience accumulée au cours du temps, la maturité, est précisément ce dont une personne âgée dispose en abondance. Le truc est de ralentir, de manière que ce capital d'expérience puisse être contemplé et, plus que cela, savouré.»

Entre autres sujets, il traite de sexualité et d'attachement conjugal. Mais aussi de dépression, et de l'ennui, facette fréquente de la vieillesse qui peut être combattue par le jeu. Mettre délibérément un terme à sa vie? Il en débat en citant les auteurs, tels que le confucéen Mencius, Sénèque bien sûr et Montaigne, pour qui la condition que la vie «ait un sens» passe avant l'idée de la prolonger autant que possible ${ }^{3}$. Il tend à être de l'avis de ces derniers et évoque la Hollande, pays de sa femme, où l'assistance au suicide est possible.

Dans un chapitre intitulé "On the timeliness of spirituality», Klein, juif non pratiquant, parle d'aspiration à la transcendance et note que, dans plusieurs religions, la spiritualité de la personne âgée a une place particulière. Il est intéressé, sent une envie (un besoin?): «Je ne m'attends pas à voir la face de Dieu ou le paysage du paradis. Ce que je recherche, c'est quelque chose comme une compréhension sublime, un accord existentiel avec l'univers; pas une chose, mais une expérience spirituelle.» Et, faisant référence à l'intérêt actuel pour la pratique dite de la pleine conscience (mindfulness): «Peut-être que ma meilleure chance de trouver la réponse à mes aspirations est d'être ici et maintenant pleinement ici et maintenant.» 